\title{
A theoretical study of serrated leading edges in aerofoil and vortical gust interaction noise
}

Xun Huang ${ }^{1,2}$

\section{Correspondence:}

huangxun@pku.edu.cn;

huangxun@ust.hk

'State Key Laboratory of Turbulence and Complex Systems, Aeronautics

and Astronautics, College of

Engineering, Peking University,

Beijing 100871, China

2Department of Mechanical and

Aerospace Engineering, The Hong

Kong University of Science and

Technology, Hong Kong, China

\begin{abstract}
Serrated leading edges are one of the most promising passive aerodynamic control methods for the reduction of aerofoil-turbulence interaction noise. To elucidate the possible physical mechanisms, the current paper studies the simplified set-up with aerofoil-vortical gust interaction and proposes an analytical model by incorporating Fourier transform into the Wiener-Hopf method. The proposed model suggests that the serrations operate on the incident vortical gusts as convolution, which leads to the innovative concept that models serrations as transfer functions in the wavenumber domain. Overall, the current theoretical study could provide a unique insight of the inherent aerodynamic noise control mechanisms of leading-edge serrations.
\end{abstract}

Keywords: Aeroacoustics, Aerofoil-turbulence interaction, Wiener-Hopf method

\section{Introduction}

Aerofoil-turbulence interaction is one of the dominant broadband flow-induced noise sources in aircraft, aeroengine and wind turbines [1-3]. Serrated leading edge, which is one of the most promising passive aerodynamic control methods, has recently received increasing research interests $[1,4,5]$. The serration approach has a presumed connection to the silent flying capability of owls [6-8] and, as shown in Fig. 1, the primary feather from an owl actually contains very long, curved and comb-like leading- and trailingedge serrations, which demands further studies of shape optimisations and modeling capabilities.

Currently, most studies of trailing-edge noise control have mainly focused on sawtoothshaped serrations [9-12], while studies of leading-edge serrations have considered potential aerodynamic drag penalties and primarily focused on wavy (sinusoidal) profiles of protuberances and tubercles [1, 4, 13]. More specifically, [5] has conducted experimental studies of a leading edge with curved sawtooth-shaped serrations and reached the conclusion that a serration with the largest amplitude (tip-to-root length) and the largest wavelength (period) is optimal. Kim et al. [1] and Zhong et al. [3] have conducted computational studies by using Euler-equations-based model with injected synthetic turbulence. The work [3] has computationally studied the interaction between the streamwise/transverse turbulent disturbances and a straight leading edge in transonic flows and shown the significantly influenced sound propagation in the presence of shocks. The

(c) The Author(s). 2019 Open Access This article is distributed under the terms of the Creative Commons Attribution 4.0 International License (http://creativecommons.org/licenses/by/4.0/), which permits unrestricted use, distribution, and reproduction in any medium, provided you give appropriate credit to the original author(s) and the source, provide a link to the Creative Commons license, and indicate if changes were made. 


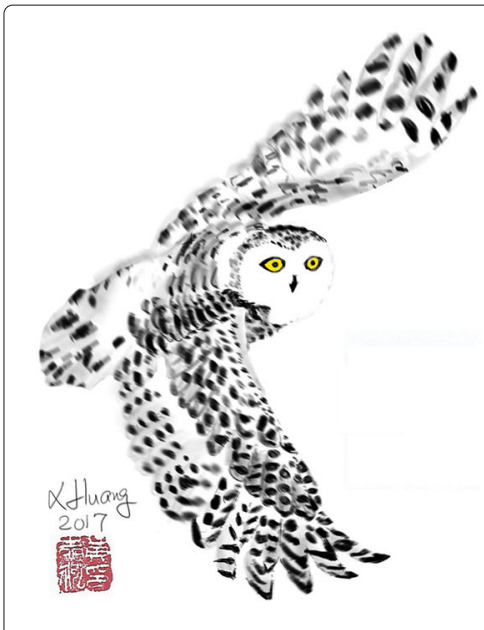

a

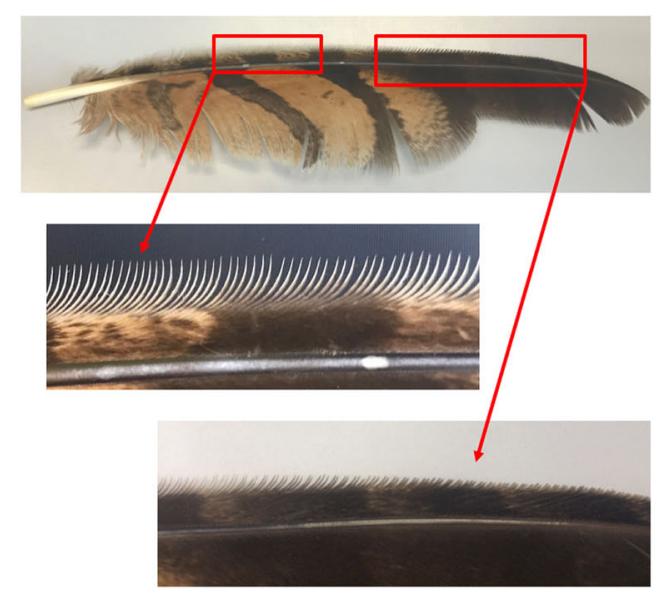

b

Fig. 1 The problem of interest, where (a) the sketch of a flying snowy owl, and (b) one of the primary feathers from an eagle owl and the corresponding leading-edge image

work [1] has simulated aerofoil-turbulence interaction noise associated with wavy leading edges in low subsonic flows and found reduced levels of radiated sound pressure due to geometric obliqueness and phase interferences.

Overall, many recent works, such as those from the groups at Brunel University and Southampton University, have performed a series of pioneering experimental studies of aerofoil aerodynamic noise with serrations in realistic set-ups $[1,5,12,14,15]$, which have provided sufficient physical insights that shall further enable the follow-up theoretical studies. Then, the main focus of the current paper is the theoretical development of an analytical model for the scattered sound waves from an infinitely thin aerofoil with serrated leading edge in the presence of a uniform background flow.

A theoretical model could enable rapid parametric studies and offer clear physical insights after taking reasonable simplifications and justifiable assumptions. To enable theoretical developments, an aerofoil is usually modeled as an infinite half plane with leading-edge serrations in the presence of uniform flows, and the incident turbulent flow is approximated by synthetic turbulence or simply a couple of eddies. By adopting such simplifications, the work [13] has developed an analytical model based on the modified Green's function from [16] and found that it is still difficult to predict the optimal serration design due to nonlinear interactions between eddies. Other than extending Howe's approach, [17] has adopted their previous approach [11] that combines coordinate transformation, Fourier series expansion and Schwarzschild techniques to propose an analytical model for leading-edge serrations. On the other hand, [18] has adopted the Wiener-Hopf method to study the effect of leading-edge radius on gust and aerofoil interaction noise, and then incorporate non-orthogonal coordinate transformation into the Wiener-Hopf model for leading-edge serrations [19] as well as trailing-edge serrations [20]. All these theoretical studies have identified some limitations. For example, the work [13] has adopted the slender body approximation from [16], which shall be unfit for long and comb-like serrations (see Fig. 1b). The references [17] and [19] have adopted coordinate transformation, which shall be most applicable to serrations with straight profiles. 
Furthermore, near-field solutions, which shall clearly illuminate the associated aerodynamic noise control mechanisms, have not been given in [19] for unknown reason. To address these issues, the current paper proposes a theoretical model by incorporating Fourier transform into the Wiener-Hopf model.

The reference [21] has incorporated Fourier series expansion into the Wiener-Hopf method to study the scattering of external sound waves due to serrations, and further extended that model for the study of Tollmien-Schlichting pressure wave scattering from serrated trailing-edge set-ups [10]. As argued by [19], our models are inevitably mathematically complicated. The current paper would endeavour to simplify our previous model by incorporating Fourier transform into the Wiener-Hopf method. The WienerHopf method is a powerful mathematical tool and has been heavily used for two types of classical wave problems in fluid mechanics: duct radiations [22-26] and flat plate diffractions $[10,18,21,27]$. The current problem falls into the second type, which usually has a much simpler Wiener-Hopf kernel than duct radiation problems. However, sound radiations from a duct are always periodic in the azimuthal direction, whereas the scattered field from a serrated flat plate could be aperiodic in the spanwise direction, which requests delicate manipulations in Fourier series expansion $[10,11]$ that however result in complicated models. Instead of using Fourier series, the current work utilises Fourier transform to establish the closed-form solution and, from which, proposes a transfer function in the chordwise-spanwise wavenumber domain to clearly elucidate the possible noise control effect of the serrations.

\section{Theoretical model}

\subsection{Description of the problem}

Figure 2 shows the current problem set-up, which contains a semi-infinite aerofoil with an infinite number of periodic rigid leading-edge serrations in the presence of a uniform flow. An incident synthetic turbulence usually consists of hundreds of Fourier modes and

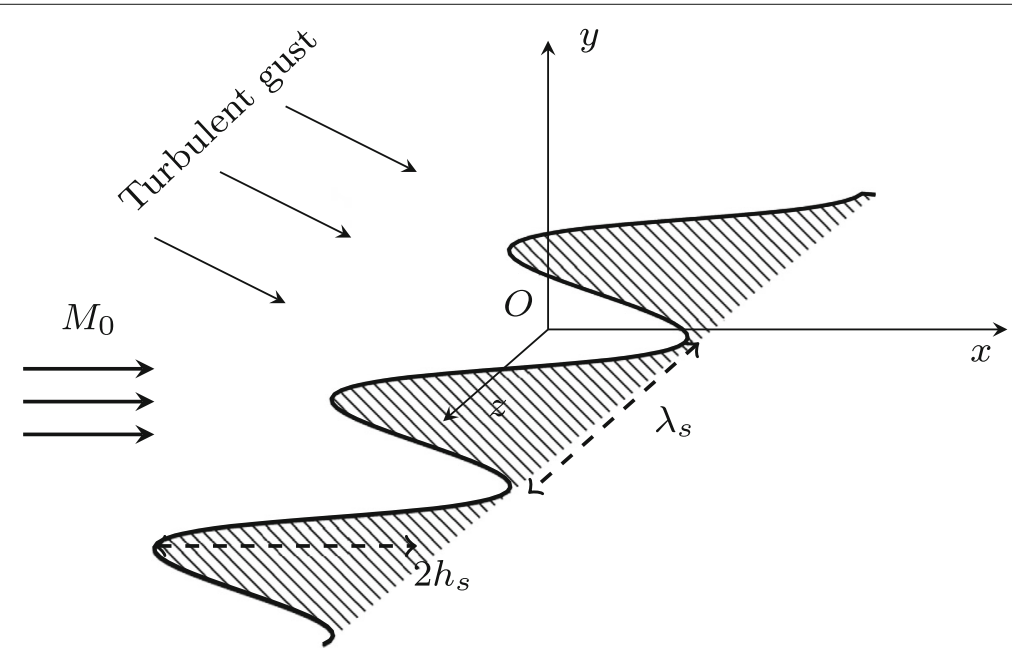

Fig. 2 Sketch of the model problem, where the (sinusoidal-shaped) wavy serrations with the profile $\chi(z)$ (with amplitude $2 h_{s}$ and the wavelength $\lambda_{s}$ ) are shown as an example. We wish to mention that our model also works for other shapes, such as sawtooth- and slotted-shaped profiles 
the associated velocity perturbation is given as the curl of a vector potential. For each single eddy case, the associated pressure boundary condition (e.g., (38) in [13]) is similar to that of an inject harmonic gust, which is

$$
v_{g}(x, y, z, t)=a_{g}(z) e^{i k_{x} x-i \omega t}, \quad \omega=k_{x} M_{0}=k,
$$

where $v_{g}$ is the velocity in $y$ and would be only meaningful for the current set-up at $y=0$, $a_{g}(z)$ is the associated amplitude with respect to $z, k_{x}$ is the normalised wavenumber in the chordwise direction, $\omega$ and $k$ are normalised angular frequency and wavenumber, and $M_{0}$ is the freestream Mach number. The associated scattered sound field from the leading edge of an aerofoil at zero angle of attack is governed by

$$
\left(\frac{\partial^{2}}{\partial x^{2}}+\frac{\partial^{2}}{\partial y^{2}}+\frac{\partial^{2}}{\partial z^{2}}\right) \psi_{s}-\left(-i k+M_{0} \frac{\partial}{\partial x}\right)^{2} \psi_{s}=0,
$$

where $\psi_{s}$ is the acoustic potential and all of the variables are non-dimensionalised using appropriate reference scales. Given $\psi_{s}$, the scattered sound pressure is $p_{s}=(-i k+$ $\left.M_{0} \partial / \partial x\right) \psi_{s}$. Following the assumption of frozen turbulence, the unsteady incident gust remains its form after convecting past the leading edge, and will then be annihilated by the scattered sound fields from the aerofoil; in addition, the jump in the scattered field across $y=0$ and $x<\chi(z)$ must be zero to satisfy the far-field radiation condition and the continuity of the scattered field upstream of the leading edge, which lead to the corresponding boundary conditions:

$$
\begin{array}{rr}
\left.\frac{\partial \psi_{s}(x, y, z)}{\partial y}\right|_{y=0}=-a_{g}(z) e^{i k_{x} x}, & \forall x \geq \chi(z), \\
\psi_{s}(x, 0, z)=0, & \forall x<\chi(z),
\end{array}
$$

where $\chi(z)$ describes the profiles of serrations. The scattered field should also satisfy the far-field radiation condition.

\subsection{The Wiener-Hopf model}

The essential concept behind this work is the incorporation of Fourier transform into the Wiener-Hopf method. First, we define the two-dimensional Fourier transform in the $(x, z)$ domain as

$$
\psi(\alpha, y, \beta)=\int_{-\infty}^{\infty} \int_{-\infty}^{\infty} \psi_{s}(x, y, z) e^{i \alpha x} e^{i \beta z} \mathrm{~d} x \mathrm{~d} z
$$

Substituting (5) into (2) yields

$$
\frac{\partial^{2}}{\partial y^{2}} \psi(\alpha, y, \beta)-\underbrace{\left(\alpha^{2}+\beta^{2}-\left(k-M_{0} \alpha\right)^{2}\right)}_{\gamma^{2}} \psi(\alpha, y, \beta)=0,
$$

where $\gamma=\gamma_{-} \gamma_{+}$and $\gamma_{ \pm}=\left[\alpha \pm\left(\left(k-M_{0} \alpha\right)^{2}-\beta^{2}\right)^{1 / 2}\right]^{1 / 2}$, from which the branch points will possibly coincide at the origin when $\beta=k$ and $M_{0}=0$, which will prevent the definitions of upper- and lower analytic half-planes. Hence, for mathematical rigor, we should mention that the current method cannot model the singular case with $\beta=k$ at $M_{0}=0$. Physically, $M=0$ means there is no flow and the gusts (or eddies, turbulence) is not convected, and no distortion happens to produce sound. By following [22], the principal branches of the square roots are chosen for $\gamma$ so that the branch cuts of $\gamma_{ \pm}$are from 
$b_{0}^{ \pm}=k /\left(M_{0} \pm 1\right)$ to $\pm \infty$, respectively, when $\beta$ is simply zero. If $\beta \neq 0$, the stating points $b_{0}^{ \pm}$would change along with $\beta$. Figure 3 shows the two half-planes and the integral path.

To satisfy the acoustic radiation condition, the solutions of (6) should have the form:

$$
\psi(\alpha, y, \beta)=\left\{\begin{array}{l}
A(\alpha, \beta) e^{-\gamma(\alpha, \beta) y}, \forall y>0 \\
-A(\alpha, \beta) e^{\gamma(\alpha, \beta) y}, \forall y<0
\end{array}\right.
$$

From (7), we can further have the following relation at $y=0^{+}$:

$$
\gamma(\alpha, \beta) \psi\left(\alpha, 0^{+}, \beta\right)+\psi^{\prime}\left(\alpha, 0^{+}, \beta\right)=0,
$$

where $(\cdot)^{+}$denotes the upper side of $(\cdot)$, and $(\cdot)^{\prime}$ represents $\mathrm{d} / \mathrm{d} y$. Next, we decompose $\psi$ into the sum of the following two parts:

$$
\begin{aligned}
& \psi(\alpha, y, \beta)=\underbrace{\int_{-\infty}^{\infty} \int_{\chi(z)}^{\infty} \psi_{s} e^{i \alpha x} e^{i \beta z} \mathrm{~d} x \mathrm{~d} z}_{=\psi_{+}(\alpha, y, \beta)}+\underbrace{\int_{-\infty}^{\infty} \int_{-\infty}^{\chi(z)} \psi_{s} e^{i \alpha x} e^{i \beta z} \mathrm{~d} x \mathrm{~d} z}_{=\psi_{-}(\alpha, y, \beta)} \\
& =\underbrace{\mathfrak{F}\left[e^{i \alpha \chi}(z)\right]}_{=\zeta(\alpha, \beta)} *(\underbrace{\mathfrak{F}\left[\int_{0}^{\infty} \hat{\psi}_{s}(\hat{x}, y, z) e^{i \alpha \hat{x}} \mathrm{~d} \hat{x}\right]}_{=\hat{\psi}_{+}(\alpha, y, \beta)}+\underbrace{\mathfrak{F}\left[\int_{-\infty}^{0} \hat{\psi}_{s}(\hat{x}, y, z) e^{i \alpha \hat{x}} \mathrm{~d} \hat{x}\right]}_{=\hat{\psi}_{-}(\alpha, y, \beta)})
\end{aligned}
$$

where $\hat{x}=x-\chi(z)$, the subscripts $(\cdot)_{ \pm}$denote analytic on the upper and lower half-planes $R_{ \pm}$, respectively, and the symbol $\mathfrak{F}[(\cdot)]$ represents the Fourier transform of $(\cdot)$ in the $z$ direction, i.e., $\mathfrak{F}[(\cdot)]=\int_{-\infty}^{\infty}(\cdot) \exp (i \beta z) \mathrm{d} z$, and $*$ represents the convolution operation.

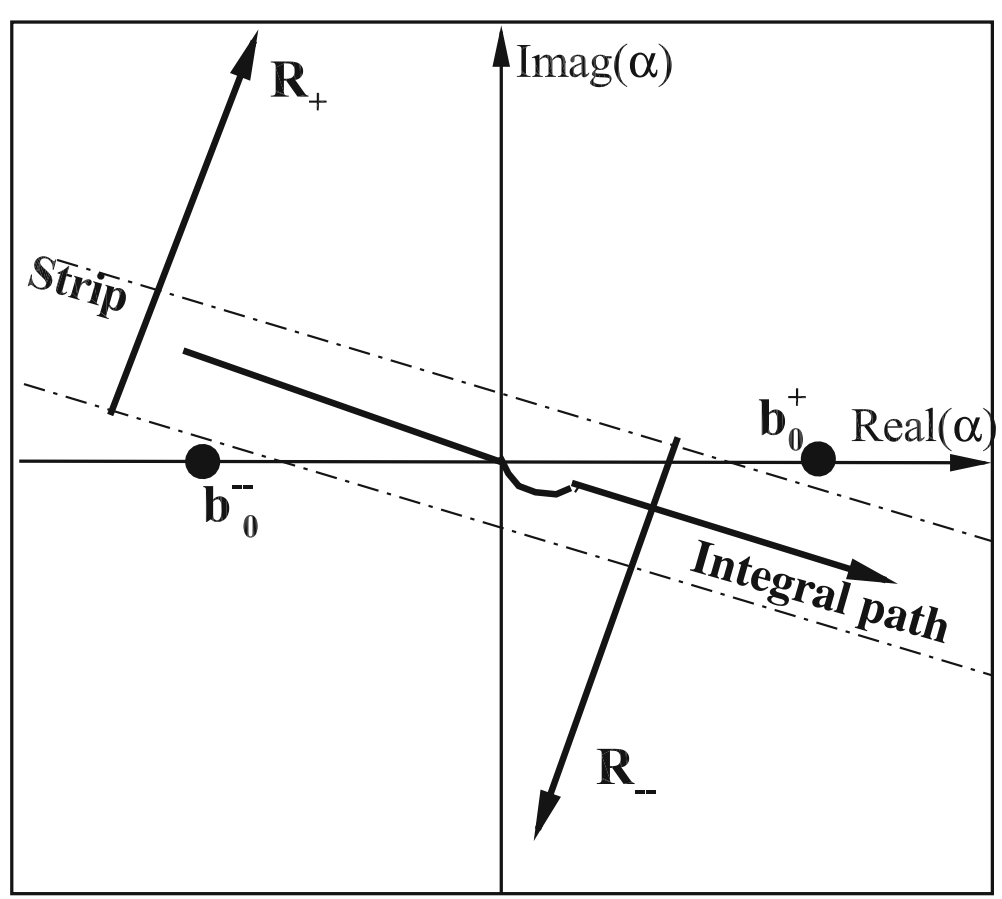

Fig. 3 Sketch of the Argand diagram of the two half-planes $R_{ \pm}$and the overlapped strip. Two branch cuts joining the branch points $b_{0}^{ \pm}$to $\pm \infty$ through the corresponding two half-planes. The integral path would move back to the horizontal axis [22] except for a slight distortion around the origin to avoid any possible singular point 
Substituting (9) into (8) yields

$$
\gamma(\alpha, \beta)(\psi_{+}\left(\alpha, 0^{+}, \beta\right)+\underbrace{\psi_{-}\left(\alpha, 0^{+}, \beta\right)}_{\equiv 0 \text { from }(4)})+\psi_{+}^{\prime}\left(\alpha, 0^{+}, \beta\right)+\psi_{-}^{\prime}\left(\alpha, 0^{+}, \beta\right)=0 .
$$

In addition, from (3), we can analytically represent

$$
\begin{aligned}
\psi_{+}^{\prime}\left(\alpha, 0^{+}, \beta\right) & =\int_{-\infty}^{\infty} \int_{\chi(z)}^{\infty}-a_{g}(z) e^{i k_{x} x} e^{i \alpha x} e^{i \beta z} \mathrm{~d} x \mathrm{~d} z \\
& =\frac{1}{i\left(\alpha+k_{x}\right)} \underbrace{\int_{-\infty}^{\infty} a_{g}(z) e^{i \beta z} \mathrm{~d} z}_{=A_{g}(\beta)} * \underbrace{\int_{-\infty}^{\infty} \underbrace{e^{i\left(\alpha+k_{x}\right) \chi(z)}}_{=\mathfrak{s}(\alpha, z)} e^{i \beta z} \mathrm{~d} z}_{=\mathfrak{S}(\alpha, \beta)},
\end{aligned}
$$

where $\mathfrak{s}(\alpha, z)$ and $\mathfrak{S}(\alpha, \beta)$ are Fourier transform pairs and solely determine the reduction effect of scattered noise due to leading-edge serrations. In addition, it is worthwhile to emphasise again that $(*)$ represents convolution in the above formulation. Mathematically, if $\chi(z)>0$, the term with $\exp (i \alpha \chi(z))$ would grow exponentially in the lower half-plane. Similarly, when $\chi(z)<0$, the term with $\exp (i \alpha \chi(z))$ would grow exponentially in the upper half-plane. Such algebraic behaviours at infinity in the upper and lower halves of the complex $\alpha$-plane are undesirable for the following procedure of the WienerHopf method. Furthermore, such infinity growth would violate the Dirichlet conditions (for Fourier expansion) and thus prevents Fourier series expansions. Our previous models $[10,21]$ avoided this critical issue by canceling out all those terms from the final WienerHopf equation. In this work, we further suggest that a weak expansion be considered for $\exp (i \alpha \chi(z))$.

Then, for periodic serrations, $\mathfrak{s}(\alpha, z)$ can be further represented by the following Fourier series,

$$
\mathfrak{s}(\alpha, z)=e^{i\left(\alpha+k_{x}\right) \chi(z)}=\sum_{\kappa=-\infty}^{+\infty} s^{[\kappa]}(\alpha) e^{i \kappa \frac{2 \pi}{\lambda_{s}} z}, s^{[\kappa]}(\alpha)=\frac{1}{\lambda_{s}} \int_{0}^{\lambda_{s}} \mathfrak{s}(\alpha, z) e^{-i \kappa \frac{2 \pi}{\lambda_{s}} z} \mathrm{~d} z,
$$

where $\lambda_{s}$ is the period of the serrations in $z$-direction. Then, (11) becomes

$$
\psi_{+}^{\prime}=\frac{A_{g}(\beta)}{i\left(\alpha+k_{x}\right)} * \sum_{\kappa=-\infty}^{+\infty} s^{[\kappa]}(\alpha) \delta\left(\beta-\kappa \frac{2 \pi}{\lambda_{s}}\right)=\frac{\sum_{\kappa=-\infty}^{+\infty} s^{[\kappa]}(\alpha) A_{g}\left(\beta-\kappa \frac{2 \pi}{\lambda_{s}}\right)}{i\left(\alpha+k_{x}\right)}
$$

where $\delta$ is the Dirac delta function. Substituting (13) into (10) results in

$$
\underbrace{\gamma_{+}(\alpha, \beta) \psi_{+}\left(\alpha, 0^{+}, \beta\right)}_{R_{+}}+\underbrace{\frac{1}{\gamma_{-}(\alpha, \beta)}}_{R_{-}} \underbrace{\psi_{+}^{\prime}\left(\alpha, 0^{+}, \beta\right)}_{\text {Pole in } R_{-}}+\underbrace{\frac{1}{\gamma_{-}(\alpha, \beta)} \psi_{-}^{\prime}\left(\alpha, 0^{+}, \beta\right)}_{R_{-}}=0,
$$

where $R_{ \pm}$in the underbrace symbol denotes the corresponding analytic half-planes, and the multiplicative factorisation [28] of $\gamma=\gamma_{+} \gamma_{-}$is imposed. We further remove the pole at $\alpha=-k_{x}$ and re-arrange (14) to 


$$
\begin{aligned}
& \underbrace{\frac{1}{\gamma_{-}(\alpha, \beta)} \psi_{-}^{\prime}\left(\alpha, 0^{+}, \beta\right)}_{R_{-}}+\underbrace{\left(\frac{1}{\gamma_{-}(\alpha, \beta)}-\frac{1}{\gamma_{-}\left(-k_{x}, \beta\right)}\right) \frac{\sum_{\kappa=-\infty}^{+\infty} s^{[\kappa]}(\alpha) A_{g}\left(\beta-\kappa \frac{2 \pi}{\lambda_{s}}\right)}{i\left(\alpha+k_{x}\right)}}_{R_{-}}= \\
& -\underbrace{\gamma_{+}(\alpha, \beta) \psi_{+}\left(\alpha, 0^{+}, \beta\right)}_{R_{+}}-\underbrace{\frac{1}{\gamma_{-}\left(-k_{x}, \beta\right)}}_{R_{+}} \underbrace{\frac{\sum_{\kappa=-\infty}^{+\infty} s^{[\kappa]}(\alpha) A_{g}\left(\beta-\kappa \frac{2 \pi}{\lambda_{s}}\right)}{i\left(\alpha+k_{x}\right)}}_{R_{+}} \equiv \mathfrak{E} .
\end{aligned}
$$

The left- and right-hand sides of (15) should be regular on the positive and negative half-planes $R_{ \pm}$, respectively. According to analytical continuation in the complex analysis theory, $\mathfrak{E}$ is an entire function that is regular on the whole complex plane. For leadingedge set-ups, $\psi_{s}(x, 0, z)$ is in the order of $x^{1 / 2}$ when $x$ approaches $\chi(z)$, which leads to $\psi(\alpha) \sim \alpha^{-1 / 2}$ when $|\alpha| \rightarrow \infty$ by using Abelian theorem [28]. Since $\psi(\alpha)=\psi_{+}(\alpha)+$ $\psi_{-}(\alpha)$, where $\psi_{-}(\alpha) \equiv 0, \psi(\alpha) \sim \alpha^{-1 / 2}$ is equivalent to $\psi_{+}(\alpha) \sim \alpha^{-1 / 2}$. In addition, from (6), it is easy to see that $\gamma_{+}(\alpha, \beta) \sim \alpha^{1 / 2}$, which leads to $\gamma_{+} \psi_{+} \sim \alpha^{0}$ and, finally, $\mathfrak{E}(\alpha) \sim \alpha^{0}$ as $|\alpha| \rightarrow \infty$. By using the extended form of Liouville's theorem [28], it is then easy to show that $\mathfrak{E}(\alpha)$ is a constant for all real $\alpha$. In this letter, we only consider the simplest scenario with the constant that equals zero. Such a simplification may be not mathematically strict but could extensively simplify the following derivations and, therefore, help to elucidate the most essential flow physics. Furthermore, a final extension to scenarios with non-zero constants shall be straightforward.

Next, by equating the right-hand side of (15) to zero, we immediately have

$$
\begin{aligned}
\psi\left(\alpha, 0^{+}, \beta\right) & =A(\alpha, \beta)=\psi_{+}\left(\alpha, 0^{+} \beta\right)+\underbrace{\psi_{-}\left(\alpha, 0^{+} \beta\right)}_{\equiv 0 \text { from }(4)} \\
& =\underbrace{-\sum_{\kappa=-\infty}^{+\infty} s^{[\kappa]}(\alpha) A_{g}\left(\beta-\kappa \frac{2 \pi}{\lambda_{s}}\right)}_{\text {Due to serrations }} \underbrace{\frac{1}{i\left(\alpha+k_{x}\right) \gamma_{+}(\alpha, \beta) \gamma_{-}\left(-k_{x}, \beta\right)}}_{P(\alpha, \beta) \text { due to straight leading edge }} .
\end{aligned}
$$

Lau et al. [10] have given a similar analytical solution for Tollmien-Schlichting pressure wave scattering from serrated trailing edges, but by incorporating Fourier series expansion into the Wiener-Hopf method. Compared to the derivations therein (Eqs. (10)-(32)), the derivations in the current paper are more succinct.

Similarly, for straight leading edges with $\chi(z) \equiv 0$, it is easy to obtain

$$
\psi\left(\alpha, 0^{+}, \beta\right)=A(\alpha, \beta)=\underbrace{-A_{g}(\beta)}_{\text {No serration }} \underbrace{\frac{1}{i\left(\alpha+k_{x}\right) \gamma_{+}(\alpha, \beta) \gamma_{-}\left(k_{x}, \beta\right)}}_{P(\alpha, \beta) \text { due to straight leading edge }} .
$$

From (7), $\psi(\alpha, y, \beta)$ is obtained. Finally, through the inverse Fourier transform, we have the analytical solution

$$
\psi_{s}(x, y, z)=\frac{1}{4 \pi^{2}} \int_{-\infty}^{+\infty} \int_{-\infty}^{+\infty}\left(\operatorname{sgn}(y) A(\alpha, \beta) e^{-\operatorname{sgn}(y) \gamma y}\right) e^{-i \alpha x-i \beta z} \mathrm{~d} \alpha \mathrm{d} \beta .
$$

Given the above solution, we can predict the far-field acoustic solutions by either following the approximation method given in [27] or the Amiet's model in [29] and [10]. It is also straightforward to establish the relation between the acoustic power spectral density and the wavenumber spectral density of incident turbulence flows by following [11] or [10]. The related details are omitted here for brevity. 


\section{Results and discussion}

As Fig. 4 shows, the above analytical model enables us to study the effect of serrations from the perspective of control system, which is one of the key contributions of this paper. Each block in the feedforward path represents a transfer function in wavenumber domain, that is, $O(\alpha, \beta) / I(\alpha, \beta)$, where $O$ and $I$ represent the output and input of each block in the wavenumber domain. For the whole feedforward loop shown in Fig. 5, the input would be $I(\alpha, \beta)=A_{g}(\beta) / i\left(\alpha+k_{x}\right)$, that is, the $\alpha-\beta$ Fourier transform of (3). Then, from (17), the output with serrations is $O_{\text {serration }}(\alpha, \beta)=I(\alpha, \beta) \cdot S(\alpha, \beta) \cdot P(\alpha, \beta)$; while from (18), the output without serrations is $O_{\text {straight }}(\alpha, \beta)=I(\alpha, \beta) \cdot P(\alpha, \beta)$. Hence, the transfer function of serrations, $S(\alpha, \beta)$, can be inferred from $O_{\text {serration }}(\alpha, \beta) / O_{\text {straight }}(\alpha, \beta)$. As a result, given (17) and (18), the transfer function for serrations is defined as

$$
S(\alpha, \beta)=\frac{\sum_{\kappa=-\infty}^{+\infty} S^{[\kappa]}(\alpha) A_{g}\left(\beta-\kappa \frac{2 \pi}{\lambda_{s}}\right)}{A_{g}(\beta)}=\frac{\sum_{\kappa=-\infty}^{+\infty} S^{[\kappa]}(\alpha) \delta\left(\beta-\kappa \frac{2 \pi}{\lambda_{s}}\right) * A_{g}(\beta)}{\delta(\beta) * A_{g}(\beta)},
$$

which shall clearly elucidate the noise control effect of the serrations. More specifically, the transfer function (20) shows that serrations operate on incident gusts (or more generally, turbulence flows) in the form of convolution. It is then straightforward to sketch a block diagram of the whole aerofoil-gust flow system and the associated passive control and optimisation procedure (see Fig. 4). We believe that (20) has some sort of connection to the so-called optical transfer function in optical systems, and (20) is not a proper transfer function in classical control since the input $A_{g}(\beta)$ cannot be easily isolated. Hence, the following study will focus on the key component $\mathfrak{S}(\alpha, \beta)$ inside $S(\alpha, \beta)$ (it should be noted that $S(\alpha, \beta) \neq \mathfrak{S}(\alpha, \beta))$.

Figure $5 \mathrm{a}$-b show the amplitude of $\mathfrak{S}(\alpha, \beta)$ without and with wavy serrations, respectively. First, (7) suggests that the scattered field becomes evanescent (i.e., source cutoff in [1]) when $\gamma^{2} \geq 0$, i.e. $\alpha^{2}+\beta^{2}-\left(k-M_{0} \alpha\right)^{2} \geq 0$, which can be approximated by $\alpha^{2}+\beta^{2} \geq k^{2}$ when $M_{0}$ is small. Here $k$ equals the nondimensional $\omega$. In other words, when the amplitude of $A(\alpha, \beta)$ is nonzero outside the circle, the associated scattered wave component becomes evanescent, and when the amplitude of $A(\alpha, \beta)$ is nonzero inside the circle, the associated scattered wave component is progressive. Then, we call the region outside the circle (with $\alpha^{2}+\beta^{2}=k^{2}$ ) as the evanescent region, and call the region inside the circle as the progress region.

For straight edges with $\chi(z)=0$, it is straightforward to get $S(\alpha, \beta) \equiv 1, \forall(\alpha, \beta)$. Then, the vortical gust at $\alpha=k_{x}, \forall \beta$ (see Fig. 5a) will be consistently scattered by the

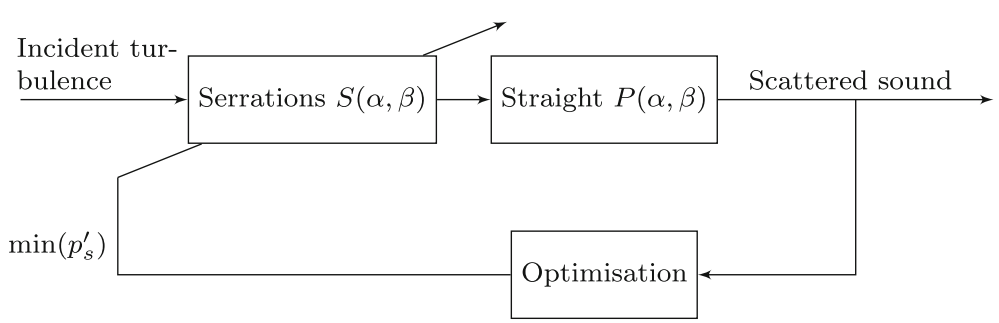

Fig. 4 The block diagram with the transfer functions in the wavenumber domain, where the variable $S(\alpha, \beta)$ is represented with the arrow, which is a common practice in classical control. In addition, various optimisation strategies can be considered and one example can be found in [33] ((2.10) therein) 


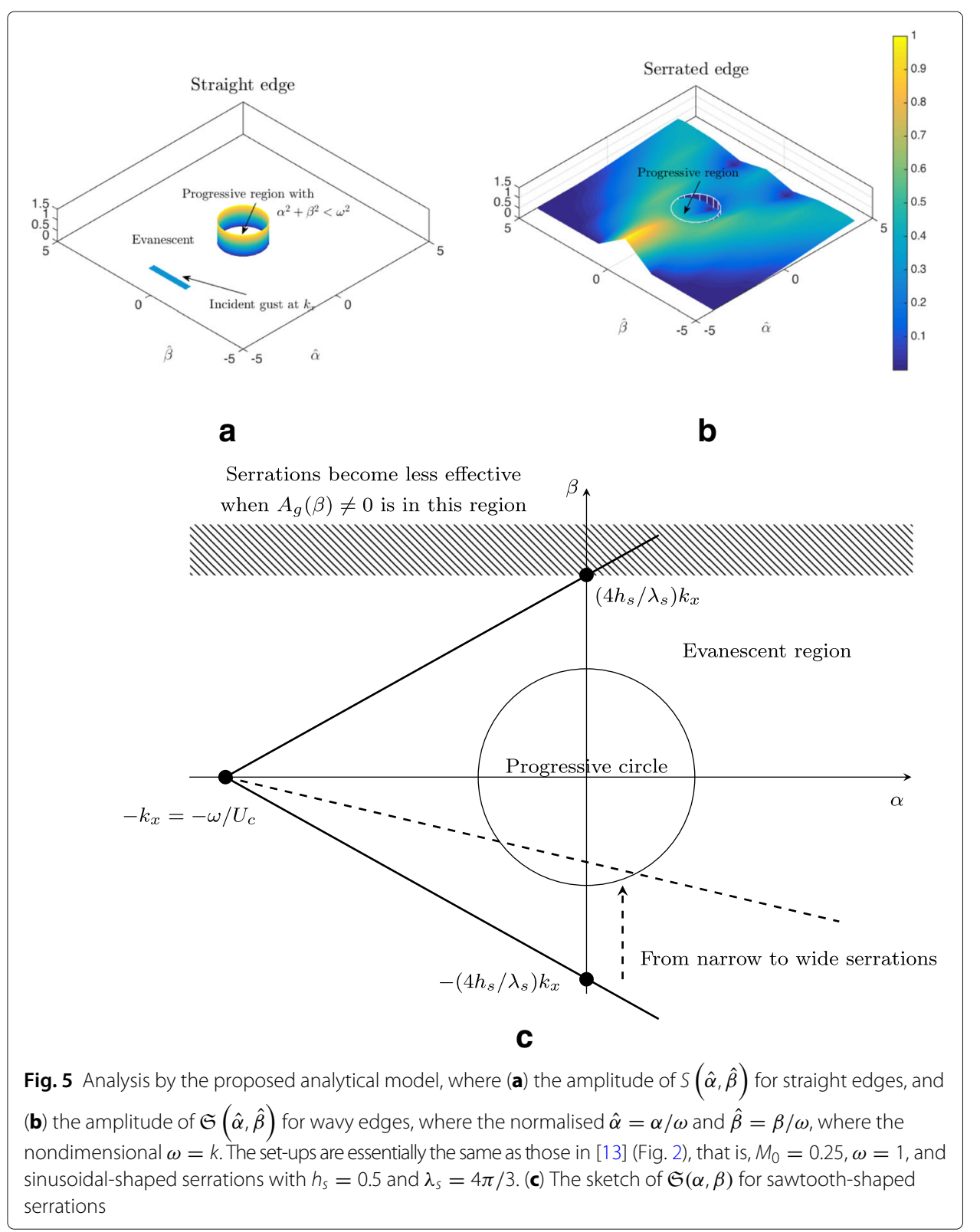

straight edge (as mathematically described by $P(\alpha, \beta)$ ) and those fall in the circle will radiate to the far field. For serrated edges, it is usually difficult to separate the incident component $A_{g}(\beta)$ from $S(\alpha, \beta)$ in (20). Hence, we directly examine $\mathfrak{S}(\alpha, \beta)$ and, as an example, show the corresponding amplitude for one wavy set-up in Fig. 5b. The geometrical configurations of the serrations are the same as those in [13] for comparison purposes. Compared to the straight edge case, the value of $\mathfrak{S}(\alpha, \beta)$ for serrated leading edge extensively decreases inside the progressive region, which corresponds to the extensive reduction of the aerofoil-gust interaction noise.

Figure $5 \mathrm{~b}$ shows that the value of $\mathfrak{S}(\alpha, \beta)$ is most dominant around $\alpha=-k_{x}$ and gradually spreads to the whole wavenumber domain mainly through two ridges. Such observations can also be explained by the proposed analytical model. Take the 
sawtooth-shaped serrations as an example, where the slope of $\chi(z)$ is $\pm 4 h_{s} / \lambda_{s}$, which leads to the following approximated relation:

$$
\mathfrak{S}(\alpha, \beta)=\int_{-\infty}^{\infty} e^{i\left(\alpha+k_{x}\right) \chi(z)} e^{i \beta z} \mathrm{~d} z \sim \delta\left(\beta+\left(\alpha+k_{x}\right) \frac{ \pm 4 h_{s}}{\lambda_{s}}\right),
$$

which is shown in Fig. 5c. The above relation becomes exact when the profile of serrations approaches the sawtooth shape. It is then easy to see that narrow serrations are more preferred than wide serrations. However, the final solution of the scattered field depends on $A_{g}(\beta) * \mathfrak{S}(\alpha, \beta)$. As a result, when the incident gust is oblique and falls in the grayed region shown in Fig. 5c, the convoluted result could fall into the circle and the corresponding scattered wave components become progressive.

Last but not least, Figs. 6 and 7 show the near-field results predicted by the proposed model. The set-ups from [13] (Fig. 2 therein) are followed here for comparison purposes. Compared to the previous results in [13], the predicted sound waves from the proposed model have almost identical patterns and values across the whole domain. In this letter, this comparison serves as the validation of the current new model. Furthermore, Fig. 7 clearly shows that the serrations induce new modes the spanwise direction, which shall be one of the main mechanisms for aerofoil-turbulence interaction noise control.

\section{Conclusion}

A theoretical model has been proposed in the current paper for the study of aerofoil-gust interaction noise with leading-edge serrations. The most essential contributions are the analytical description and the transfer function concept based on Fourier transform and the Wiener-Hopf method. We wish to mention that the derivation of theoretical models is only possible by adopting justifiable simplifications and approximations. The current model employs the frozen turbulence assumption and semi-infinite thin aerofoil in the presence of uniform flows at zero angle of attack. It is well known that frozen turbulence assumption is usually valid for uniform or weak sheared flows. The semi-infinite aerofoil assumption should be valid for high-frequency gusts. It is possible to extend the current
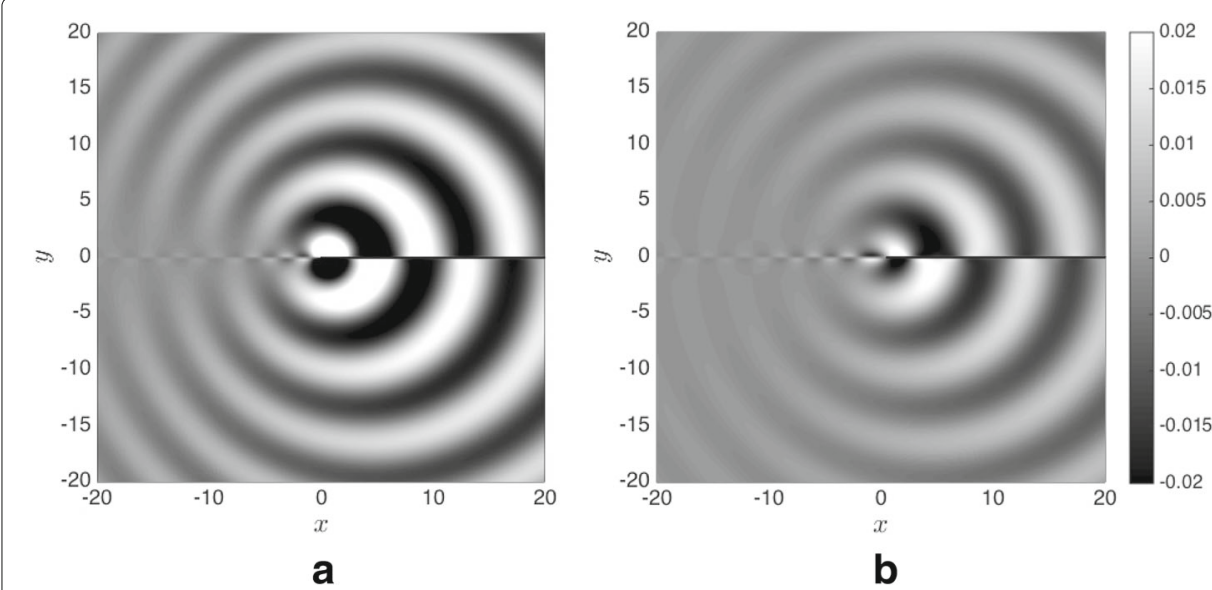

Fig. 6 The near-field results at $z=-5$ from the theoretical model and the set-ups are the same as those in Fig. 5(a) and (b), respectively. Tiny (spurious) oscillations around $(x<0, y=0)$ should not affect sound fields at the far-field 

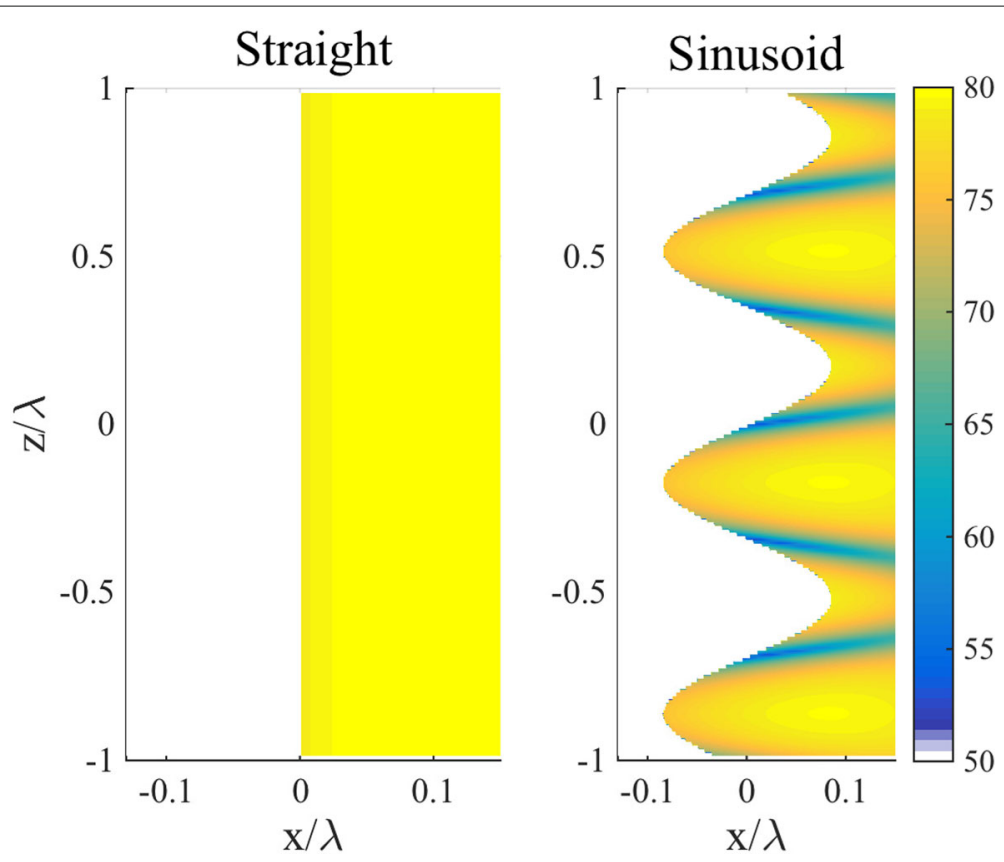

Fig. 7 The predicted sound pressure levels (in $\mathrm{dB}$ ) of the scattered sound field on the upper surface at $y=0^{+}$, where the vertical axis shows the spanwise coordinate and the horizontal axis shows the streamwise coordinate. Both coordinates are normalised by the sound wavelength at the frequency. The other set-ups are the same as those in Fig. 6

Wiener-Hopf model for finite aerofoil set-ups by using the approximate methods in [28] (Chap. V). Nonzero angle of attack effect has been analytically studied by [30] and [13] based on beautiful but complicated mathematical methods. The current work tries to provide the analytical model and the associated transfer function concept in the most succinct way and, hence, has to adopt the above simplifications and assumptions, which should hopefully retain the most important features of the serrated-aerofoil-flow system that is investigated.

The proposed model shall enable one to rapidly study the noise reduction performance of various-shaped (and even aperiodic) serrations for different incident vortical gusts. It shall be straightforward to further extend to turbulence flow set-ups. Further, the transfer function concept shall enable one to elucidate the associated physical mechanisms in a systematic way. Overall, the current model extensively simplifies our previous analytical modes for serrations $[10,21]$ and should be able to provide a clear physical insight of the inherent noise control mechanisms. In particular, the innovative concept that models the effect of serrations as transfer functions is uniquely different from other analysis in fluid mechanics and shall be able to assist the future analysis, design and optimisation of new low-noise aviation systems [2, 31, 32].

Acknowledgements

The author is grateful to Miss. Yi Fang for preparing the photo of the feather.

Funding

This work is partly supported by National Science Foundation of China (Grants 11772005, 91852201), Ministry of Industry and Information Technology of China (Grant no. MJ-2015-F-012-03) and the Research Grants Council of the Hong Kong Special Administrative Region (Grant no. 16205317). 
Availability of data and materials

The code of the basic vortex-plate interaction noise model could be shared at the request of readers.

\section{Authors' contributions}

$\mathrm{XH}$ implemented the theoretical model and conducted analysis independently. The author read and approved the final manuscript.

\section{Authors' information}

Dr. Huang received the B.Eng. degree in aerospace engineering from the Northwestern Polytechnical University, Xi'an, China, the M.Eng. degree in automatic control from Tsinghua University, Beijing, China, and the Ph.D. degree in aeronautics and astronautics from the University of Southampton, Southampton, U.K., in 1999, 2002, and 2006, respectively. Dr. Huang was a recipient of the Edison Technology Excellence Award in 2003, Award of Excellent Young Scholar from National Science Foundation of China in 2013, Newton Advanced Fellow from Royal Society UK in 2015, and AIAA Associate Fellow in 2018.

\section{Competing interests}

Not applicable.

\section{Publisher's Note}

Springer Nature remains neutral with regard to jurisdictional claims in published maps and institutional affiliations.

Received: 23 January 2019 Accepted: 30 January 2019

Published online: 28 February 2019

\section{References}

1. Kim JW, Haeri S, Joseph PF (2016) On the reduction of aerofoil-turbulence interaction noise associated with wavy leading edges. J Fluid Mech 792:526-552

2. Wang ZY, Zhuang M (2017) Leading-edge serrations for performance improvement on a vertical-axis wind turbine at low tip-speed-ratios. Appl Energy 208:1184-1197

3. Zhong SY, Zhang X, Gill J, Fattah R (2018) A numerical investigation of the airfoil-gust interaction noise in transonic flows: acoustic processes. J Sound Vib 425:239-256

4. Biedermann TM, Chong TP, Kameier F, Paschereit CO (2017) Statistical-empirical modeling of airfoil noise subjected to leading-edge serrations. AIAA J 55(9):3128-3142

5. Juknecivius A, Chong TP (2018) On the leading edge noise and aerodynamics of thin aerofoil subjected to the straight and curved serrations. J Sound Vib 425:323-343

6. Chen R, Ikeda T, Nakata T, Liu H (2017) Owl-inspired leading-edge serrations play a crucial role in aerodynamic force production and sound suppression. Bioinspiration Biomimetics 12:046008

7. Lilley GM (1998) A study of the silent flight of the owl. AIAA Paper:1998-2340

8. Lilley GM (2001) The prediction of airframe noise and comparison with experiment. J Sound Vib 239(4):849-859

9. Howe MS (1991 b) Noise produced by a sawtooth trailing edge. J Acoust Soc Am 90(1):482-487

10. Lau ASH, Huang X (2018) The control of aerodynamic sound due to boundary layer pressure gust scattering by trailing edge serrations. J Sound Vib 432:133-154

11. Lyu B, Azarpeyvand M, Sinayoko S (2016) Prediction of noise from serrated trailing edges. J Fluid Mech 793:556-588

12. Stalnov O, Chaitanya P, Joseph PF (2016) Towards a non-empirical trailing edge noise prediction model. J Sound Vib 372:50-68

13. Mathews JR, Peake N (2018) An analytically-based method for predicting the noise generated by the interaction between turbulence and a serrated leading edge. J Sound Vib 422(24):506-525

14. Narayanan S, Chaitanya P, Haeri S, Joseph P, Kim JW, Polacsek C (2015) Airfoil noise reductions through the edge serrations. Phys Fluids 27:025109

15. Vathylakis A, Chong TP, Joseph PF (2015) Poro-serrated trailing edge devices for airfoil self-noise reduction. AIAA J 53(11):3379-3394

16. Howe MS (1991a) Aerodynamic noise of a serrated trailing edge. J Fluids Struct 5:33-45

17. Lyu B, Azarpeyvand M (2017) On the noise prediction for serrated leading edges. J Fluid Mech 826:205-234

18. Ayton $\sqcup(2016 \mathrm{~b})$ Interaction of turbulence with the leading-edge stagnation point of a thin aerofoil. J Fluid Mech 798:436-456

19. Ayton $\amalg$, Kim JW (2018) An analytic solution for the noise generated by gust-aerofoil interaction for plates with serrated leading edges. J Fluid Mech 853:515-536

20. Ayton $\sqcup$ (2018) Analytic solution for aerodynamic noise generated by plates with spanwise-varying trailing edges. J Fluid Mech 849:448-466

21. Huang $X(2017)$ Theoretical model of acoustic scattering from a flat plate with serrations. J Fluid Mech 819:228-257

22. Gabard G, Astley RJ (2006) Theoretical model for sound radiation from annular jet pipes: far- and near-field solutions. J Fluid Mech 549:315-341

23. Liu X, Jiang HB, Huang X, Chen S (2016) Theoretical model of scattering from flow ducts with semi-infinite axial liner splices. J Fluid Mech 786:62-83

24. Munt RM (1977) The interaction of sound with a subsonic jet issuing from a semi-infinite cylindrical pipe. J Fluids Mech 83:609-640

25. Rienstra SW (1984) Acoustic radiation from a semi-infinite annular duct in a uniform subsonic mean flow. J Sound Vib 94(2):267-288

26. Veitch B, Peake N (2008) Acoustic propagation and scattering in the exhaust flow from coaxial cylinders. J Fluid Mech 613:275-307 
27. Ayton $\sqcup$ (2016a) Acoustic scattering by a finite rigid plate with a poroelastic extension. J Fluid Mech 791:414-438

28. Noble B (1958) Methods based on the Wiener-Hopf technique for the solution of partial differential equations. Pergamon Press:48-61

29. Roger M, Moreau S (2005) Back-scattering correction and further extensions of amiet's trailing-edge noise model. part 1: theory. J Sound Vib 286:477-506

30. Myers MR, Kerschen EJ (1995) Influence of incidence angle on sound generation by airfoils interacting with high-frequency gusts. J Fluid Mech 292:271-304

31. Ran LK, Ye CC, Wan ZH, Yang HH, Sun DJ (2018) Instability waves and low-frequency noise radiation in the subsonic chevron jet. Acta Mech Sin 34(3):421-430

32. Zhang X (2015) Aircraft noise and its nearfield propagation computations. Acta Mech Sin 28(4):960-977

33. Huang X, Zhong SY, Liu X (2014) Acoustic invisibility in turbulent fluids by optimised cloaking. J Fluid Mech 749:460-477

Ready to submit your research? Choose BMC and benefit from:

- fast, convenient online submission

- thorough peer review by experienced researchers in your field

- rapid publication on acceptance

- support for research data, including large and complex data types

- gold Open Access which fosters wider collaboration and increased citations

- maximum visibility for your research: over $100 \mathrm{M}$ website views per year

At BMC, research is always in progress.

Learn more biomedcentral.com/submissions 